\title{
1 A global meta-analysis reveals more variable life histories in urban 2 birds compared to their non-urban neighbours
}

4 Authors:

5 Pablo Capilla-Lasheras ${ }^{1 *}$, Megan J. Thompson ${ }^{2,3}$, Alfredo Sánchez-Tójar ${ }^{4}$, Yacob Haddou ${ }^{1}$,

6 Claire J. Branston ${ }^{1}$, Denis Réale ${ }^{2}$, Anne Charmantier $^{3}$, Davide M. Dominoni ${ }^{1}$

8 Affiliations:

$9{ }^{1}$ Institute of Biodiversity Animal Health \& Comparative Medicine, University of Glasgow,

10 Scotland

$11{ }^{2}$ Département des Sciences Biologiques, Université du Québec à Montréal, Canada

$12{ }^{3}$ Centre d'Ecologie Fonctionnelle et Evolutive, Université de Montpellier, CNRS, EPHE, IRD,

13 Montpellier, France

$14{ }^{4}$ Department of Evolutionary Biology, Bielefeld University, Bielefeld, 33501, Germany.

* Corresponding author: Pablo Capilla-Lasheras (pacapilla@gmail.com)

ORCID

19 PC-L: 0000-0001-6091-7089

20 MJT: 0000-0002-0279-5340

21 AS-T: 0000-0002-2886-0649

$22 \mathrm{YH}: 0000-0003-0557-0339$

23 CB: 0000-0002-4416-0984

24 DR: 0000-0002-0419-7125

25 AC: 0000-0002-0691-2647

26 DD: 0000-0003-2063-9955 


\section{Abstract.}

28 Cities pose a major ecological challenge for wildlife worldwide. Phenotypic variation is a

29 pivotal metric to predict evolutionary potential in response to environmental change. Recent

30 work suggests that urban populations might have higher levels of phenotypic variation than

31 non-urban counterparts. This prediction, however, has never been tested across taxa nor

32 over a broad geographical range. Here, we conduct a meta-analysis of the urban avian

33 literature to compare urban versus non-urban means and variances for phenology and

34 reproductive performance. First, we show that urban birds reproduce earlier and have

35 smaller broods than non-urban ones. Second, we demonstrate that urban populations have

36 a more variable phenology than non-urban populations. Our analyses reveal that the latter

37 pattern arises from differences in phenological variation between individuals within breeding

38 seasons, likely because of higher heterogeneity in the urban study areas. These findings

39 suggest that the opportunity for selection on phenology may be stronger in urban bird

40 populations and that the patterns of phenotypic variation in urban and non-urban avian

41 populations may consistently differ. 


\section{Introduction}

43 Humans have drastically changed the environmental conditions on Earth ${ }^{1}$. The footprint of

44 human activity is most pronounced in urban environments, where microclimatic conditions,

45 biogeochemical cycles and the sensory landscape are considerably different to those in non-

46 urban environments ${ }^{2}$. Perhaps not surprisingly, animals and plants are changing their

47 phenotypes in response to the novel conditions and selective pressures found in cities $^{3,4}$

48 and, indeed, numerous studies have reported contrasting phenotypes between urban and

49 non-urban populations across the globe (e.g. ${ }^{5-7}$; reviewed $\mathrm{in}^{3,4,8}$ ). The extent to which

50 populations can adapt to urban living will partly depend on how urbanisation affects

51 phenotypic variation ${ }^{9}$. Phenotypic variation is tightly linked to eco-evolutionary processes: it

52 is an essential condition for current selection, it results from past selection pressures, and it

53 depends on gene flow and phenotypic plasticity. As such, assessing how urbanisation

54 affects phenotypic variation is essential to understand the evolutionary drivers of and

55 potential for phenotypic change in urban environments.

57 Urban evolutionary ecologists have traditionally focused on differences in mean trait values

58 between urban and non-urban populations, typically finding altered phenology, morphology,

59 behaviour and reproductive performance in urban populations (or populations exposed to

60 urban-like factors) compared to non-urban counterparts ${ }^{10-14}$. However, no study to date has

61 explicitly investigated how urbanisation affects phenotypic variation at a global scale (see ${ }^{15}$

62 for a study of changes in fitness variation in response to human-related activities).

64 Recent single-species studies suggest that phenotypic variation may be affected by 65 urbanisation $^{9,16}$. For example, in species with limited dispersal ability (i.e., whose dispersal 66 occurs at a smaller scale than the scale at which the urban habitat varies), adaptation to 67 local conditions may increase phenotypic variation within the urban matrix. Findings from 68 urban and non-urban meta-populations of the common ragweed (Ambrosia artemisiifolia) are consistent with this prediction as inter-population variation in several fitness proxies was 
70 greater in urban environments compared to non-urban environments ${ }^{16}$. A recent meta-

71 analysis of selection strength found weaker selection occurring in human-disturbed

72 populations ${ }^{15}$, which could lead to higher phenotypic variation in urban populations

73 compared to their non-urban counterparts. Overall, these studies converge with the notion

74 that urban populations may display higher levels of phenotypic variation (reviewed $\mathrm{in}^{9}$ ).

75 These findings also highlight that the extent to which urbanisation may impact phenotypic

76 variation is likely to depend on the interplay between the spatial and temporal scale at which

77 environmental conditions fluctuate in the urban habitat, and the species' dispersal ability and

78 longevity 9 .

80 Here, we investigate how urbanisation impacts mean phenotypic values and phenotypic 81 variation using a meta-analysis of 409 urban - non-urban comparisons of avian life-history

82 traits (laying dates, clutch size and number of fledglings) published between 1958 and 2020

83 including 33 bird species (Figure 1). Specifically, we ask the following questions: i) does

84 urbanisation affect mean life-history avian phenotypes? ii) does urbanisation impact variation

85 in avian life-history traits? iii) what is the temporal and spatial scale at which urbanisation

86 affects phenotypic variation? Based on previous research ${ }^{10,13}$, we predict that urban bird

87 populations display earlier phenology and are less productive than non-urban populations.

88 We also predict increased phenotypic variation in urban populations compared to non-urban

89 populations (see above; and reviewed $i^{9}$ ). Lastly, we disentangle urban effects on

90 phenotypic variation across different temporal and spatial scales, suggesting an ecological

91 cause for the effects of urbanisation on avian phenotypic variation. 

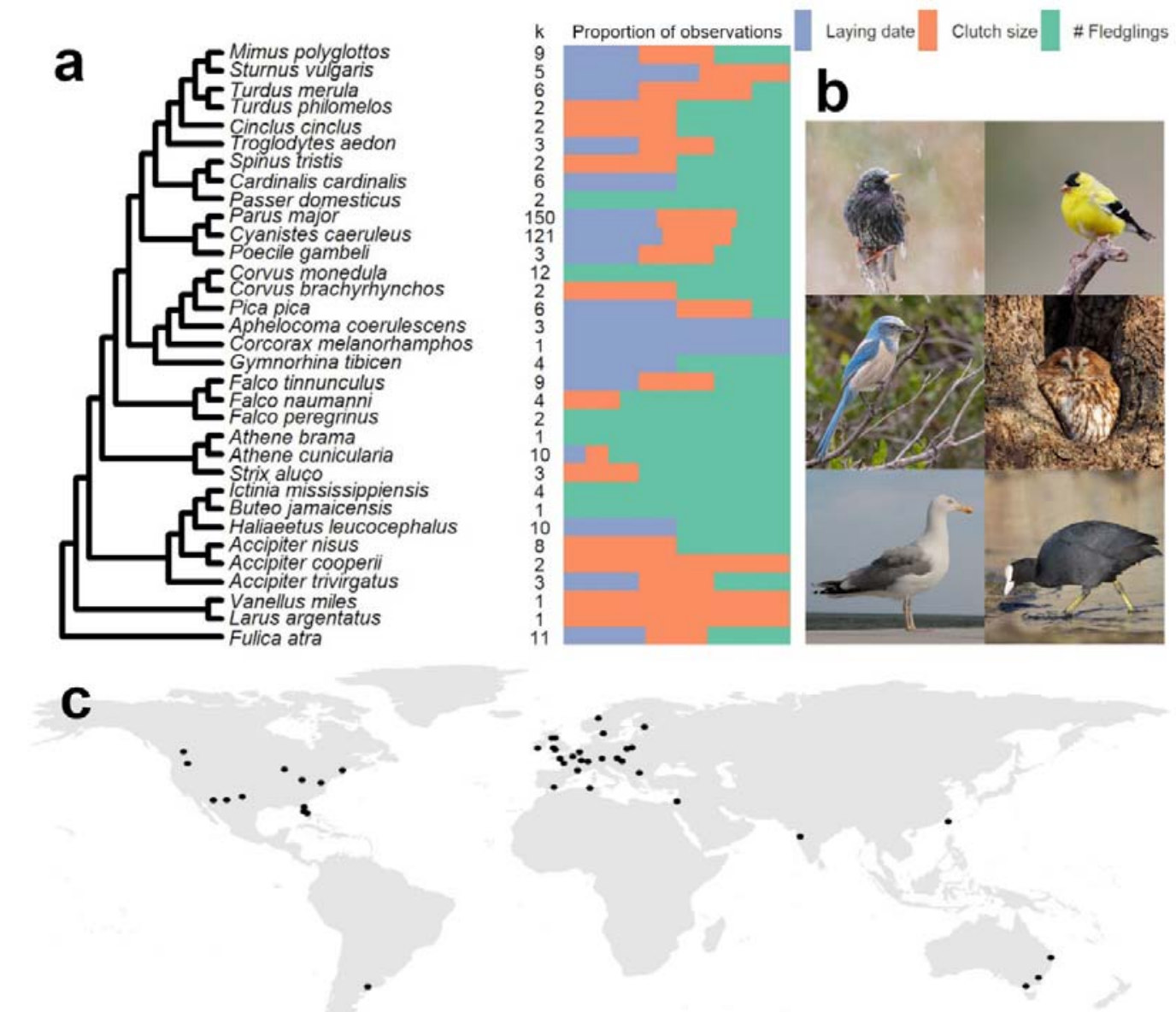

94 Figure 1. Phylogenetic and geographical breadth of the meta-analytic data. (a) Phylogenetic tree of the 33 avian species included in the meta-analysis along with the number of effect sizes (i.e., urban - non-urban comparisons) included per species ('k'; which may encompass multiple years of study from the same publication) and the proportion of observations for each life-history trait (purple: Laying date; orange: Clutch size; Green: Number of fledglings). (b) Our meta-analysis included a broad range of species, as examples, left to right from top to bottom: Sturnus vulgaris, Spinus tristis, Aphelocoma coerulescens, Strix aluco, Larus argentatus and Fulica atra. All images are copyright free (CC - Public Domain Mark 1.0. Authors: Shenandoah National Park [first two images], Mike Carlo/U.S. Fish \& Wildlife Service, Raini Svensson, Vanzan and Ekaterina Chernetsova) and were extracted from www.flickr.com. (c) Global map (excluding Antarctica) showing the location of each study included in the meta-analysis. Each point represents one study in which one or more urban - non-urban pairs of populations were sampled across a varying number of years. 


\section{Results \& Discussion}

110 After systematically inspecting 940 studies published between 1958 and 2020 (see 111 Methods), our meta-analysis included 409 urban - non-urban comparisons for three life112 history traits: laying date (151 effect sizes from 32 studies), clutch size (124 effect sizes from 11342 studies) and number of fledglings (134 effect sizes from 44 studies) (Figure 1, S1 \& S2).

114 To test whether urban populations reproduce earlier, have smaller clutches, fewer nestlings 115 survive to fledging, and display more variation in these life-history traits compared to non116 urban conspecifics, we meta-analysed log response ratios $\left(\operatorname{lnR} R^{17}\right)$ and log coefficient of 117 variation ratios $\left(\operatorname{lnCVR}{ }^{18,19}\right)$ accounting for phylogenetic effects ${ }^{20}$. These effect sizes were used to compare means and coefficients of variation of urban versus non-urban populations, and are widely used in evidence synthesis in ecology and evolution ${ }^{18,21,22}$. In the context of this meta-analysis, positive values of InRR and InCVR indicate higher mean values and higher variation, respectively, in urban populations compared to non-urban populations (and vice versa; see Methods).

Combining data across the three studied life-history traits, urban populations had overall lower mean values than their non-urban counterparts (InRR mean estimate [95\% Confidence Interval $]=-0.050[-0.096,-0.004] ; P_{\text {total }}^{2}=98.6 \%$; Figure S4; Table S1). Further analysis accounting for potential covariation across the three investigated traits in the response to urbanisation (i.e., using a model with an unstructured variance-covariance matrix; see Methods and Table S2) confirmed that urban populations had lower mean values in every 130 life-history trait: urban populations laid their eggs earlier $(\operatorname{lnRR}[95 \% \mathrm{Cl}]=-0.056[-0.084$, 131 0.028]; Figure 2a), laid smaller clutches (InRR [95\% Cl] = -0.075 [-0.109, -0.040]; Figure 2a), and tended to fledge fewer nestlings per clutch than non-urban populations (InRR $[95 \% \mathrm{Cl}]=$ $-0.075[-0.183,0.034]$; Figure $2 \mathrm{a}$ - but note that the $95 \% \mathrm{Cl}$ overlaps zero in this case, seemingly due to more variation in InRR for fledgling number than for clutch size, see Figure S6a). This meta-analytic model estimated different random effect intercepts per trait and allowed for correlations across traits (see Methods for details). This model revealed strong 
137 correlations in the response to urbanisation across traits: studies reporting large differences

138 in laying dates between urban and non-urban populations also reported small differences

139 between habitats in clutch size and number of fledglings (see 'Study ID (correlations)' in

140 Table S3; i.e., correlations among studies in the values of InRR for each trait).

142 These results indicate that urban bird populations overall lay their eggs earlier and produce 143 smaller clutches, which results in a lower number of surviving fledglings than their non-urban 144 neighbouring populations. These findings are largely in agreement with previous metaanalyses investigating the effects of urbanisation on mean avian life-histories ${ }^{10,13}$, whose conclusions we have confirmed using a larger dataset with higher spatial, temporal and phylogenetic coverage. Additionally, our results suggest the mean response to urbanisation is correlated among traits. Interestingly, we found that earlier laying dates in urban populations compared to non-urban populations were associated with smaller differences in clutch size and number of surviving nestlings between habitats (after accounting for mean differences among traits between habitats). Clutch size decreases with laying date in multiple bird species ${ }^{23}$, and these two traits are often hypothesized to co-evolve because of a strong negative genetic correlation ${ }^{24}$. All else being equal, urban conditions that advance the onset of reproduction (e.g., light pollution ${ }^{25}$ ) could indirectly increase clutch size and, therefore, reduce differences in reproductive output (clutch size and, possibly, number of fledglings) between urban and non-urban populations that arise via other mechanisms (e.g., resource limitation $\left.{ }^{26,27}\right)$.

When combining data across the three studied life-history traits, the coefficient of phenotypic variation in urban populations was $8.0 \%$ higher than in non-urban populations (InCVR mean estimate $[95 \% \mathrm{Cl}]=0.077[-0.032,0.186] ; P_{\text {total }}=71.9 \%$; Figure S5 and Table S1; note that the $95 \% \mathrm{Cl}$ overlapped zero). A subsequent model of InCVR accounting for potential covariation across the three investigated traits in the response to urbanisation (see Methods and Table S4) revealed that this trend was mainly driven by urban populations having a 
165 more variable phenology $(\operatorname{InCVR}$ mean for laying date $[95 \% \mathrm{Cl}]=0.193[0.107,0.279]$, i.e.,

$16621.9 \%$ more variation, on average, in laying date in urban than non-urban populations).

167 Urban and non-urban populations did not statistically differ in the amount of phenotypic 168 variation in clutch size or number of fledglings (i.e., 95\% Cls overlapped zero; the direction 169 of the effect reflected higher phenotypic variation in urban populations for both traits; Figure 170 2b) (InCVR mean estimates [95\% Cl]: clutch size $=0.052[-0.046,0.149]$, number of 171 fledglings $=0.064[-0.071,0.198])$. We did not find evidence for the existence of correlations 172 among life-history traits in their InCVR (models including correlations among traits scored 173 more than 1.57 AIC points below the top model, which only included independent random 174 intercepts per trait; Table S4). Differences in phenotypic variation between habitats were 175 quantitatively very similar when we meta-analysed the amount of variation (i.e., $\operatorname{lnVR}^{18}$ ) 176 instead of the coefficient of variation (i.e., InCVR; Figure S6). 

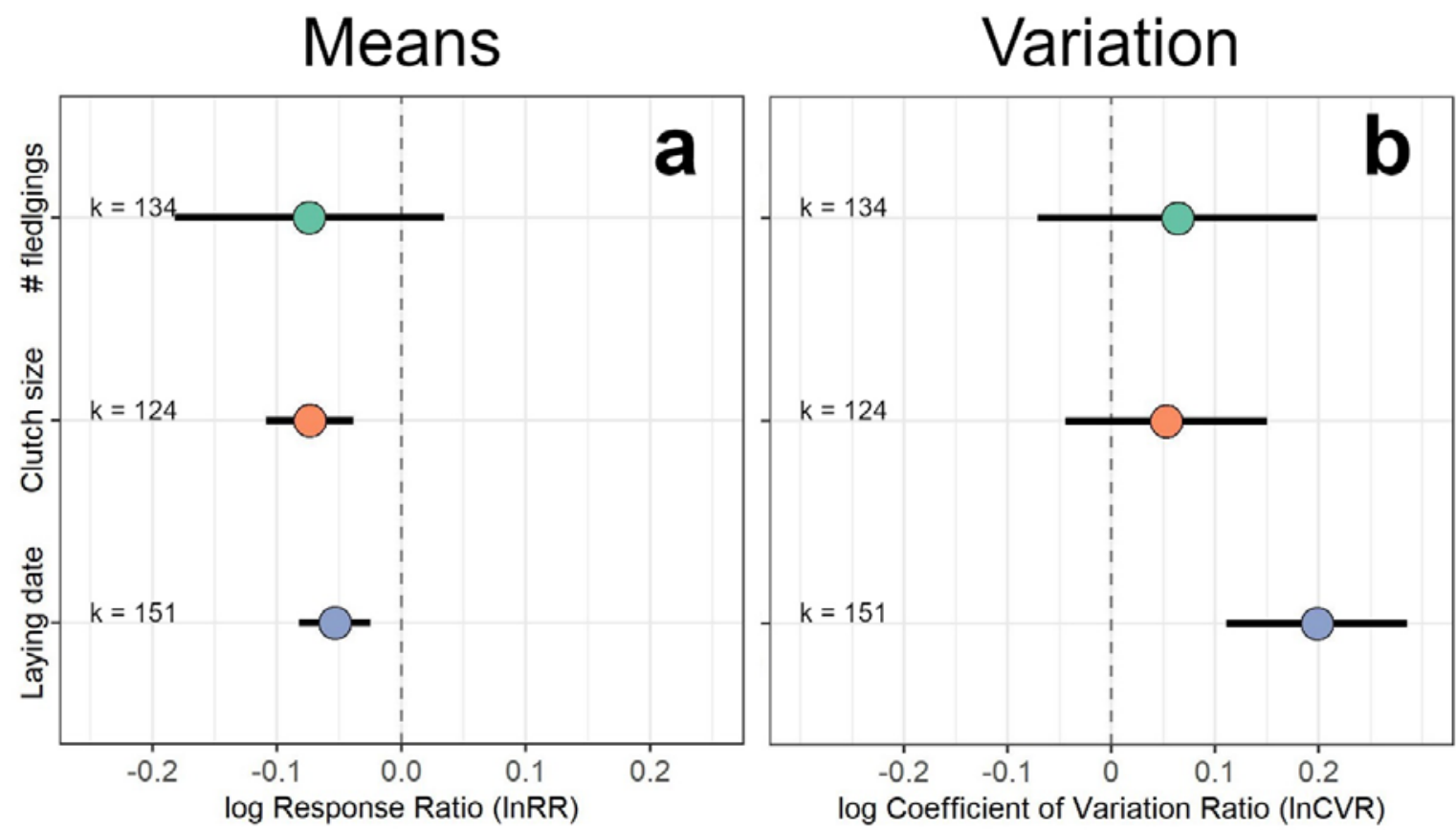

178 Figure 2. Urban populations have earlier phenology, lower reproductive output and

179 more variable life-history traits than non-urban populations. (a) Urban populations laid

180 earlier and had smaller clutches, producing fewer fledglings, than their paired non-urban

181 populations (illustrated by negative InRR estimates). (b) Our meta-analysis revealed that

182 variation in life-history traits was higher in urban populations compared to non-urban

183 counterparts, with a marked difference between habitats in laying dates (illustrated by

184 positive estimates of $\operatorname{lnCVR}$ ). Model estimates for (a) InRR and (b) InCVR are shown along

185 with their 95\% confidence intervals per trait as calculated by our meta-analytic models

186 accounting for correlated responses to urbanisation among traits (see Table S3 \& Table S5

187 for full model outputs and Figure S4 and S5 for overall meta-analyses of InRR and InCVR).

188 Raw data and model estimates are presented in Figure S6. 'k' provides the number of urban

189 - non-urban comparisons. 
191 Higher phenotypic variation in phenology in urban compared to non-urban avian populations 192 might indicate that the opportunity for selection to act on phenological traits is stronger in 193 urban populations ${ }^{28}$. However, the actual evolutionary implications of this finding are likely to depend on the interplay between the temporal scale at which urbanisation affects phenotypic variation and the longevity of a given species ${ }^{9}$. Phenotypic variation in phenology could be increased in urban bird populations by at least two temporal mechanisms acting alone or simultaneously. First, urban populations could display higher variation in phenology within a given breeding season as a result of increased spatial heterogeneity in urban habitats ${ }^{29}$. Second, urban populations could display higher variation in phenology due to larger yearly fluctuations in environmental conditions, with or without differences in phenotypic variation between urban and non-urban populations within a given breeding season. In this latter scenario, natural selection in short-lived species, whose individuals mostly breed one single year, may not act on different phenotypic variation between urban and non-urban habitats.

Additional analyses attending to the temporal source of differences in phenotypic variation for laying date between urban and non-urban populations demonstrated that these arose from differences in variation within breeding seasons rather than being the result of more variation in phenology between breeding seasons (Table 1). While laying date in urban populations was more variable than in non-urban populations within breeding seasons (InCVR mean estimate $[95 \% \mathrm{Cl}]=0.193[0.094,0.292]$; Table 1$)$, a subsequent metaanalytic model isolating effects on phenotypic variation arising from between breeding season fluctuations revealed no statistical difference between urban and non-urban populations (InCVR intercept mean $[95 \% \mathrm{Cl}]=0.007[-0.133,0.147]$; Table 1). While the sample size for this latter meta-analysis was almost four times smaller than for the metaanalysis of within breeding season differences in variation, the estimated InCVR in both models showed large differences: mean InCVR within breeding seasons was more than 27 times larger than mean InCVR among breeding seasons. 
219 Higher phenotypic variation in urban than in non-urban populations within breeding seasons

220 can be explained by at least two different processes: i) differences in the additive genetic

221 variance in laying dates, whereby urban birds have a wider range of genotypes for laying

222 dates, or ii) differences in habitat heterogeneity influencing plasticity in laying dates, whereby

223 urban areas have larger environmental variation than non-urban habitat ${ }^{30-32}$. While

224 environmental conditions are often found to be different between urban and non-urban

225 habitats $^{33}$, and this is likely to impact patterns of phenotypic variation ${ }^{7,34}$, the role of habitat

226 heterogeneity in shaping differences in phenotypic variation between urban and non-urban

227 avian populations has received little attention ${ }^{29,31}$. Our analyses indicate that differences in

228 urban versus non-urban habitat heterogeneity could indeed contribute to explain the

229 observed pattern of increased phenotypic variation in urban populations. Using a subset of

230 studies that reported the approximated location of their urban and non-urban populations, we

231 quantified habitat heterogeneity and assessed whether urban populations were exposed to a

232 more heterogeneous habitat than non-urban populations ${ }^{29}$, and whether the increase in

233 phenotypic variation in urban habitats was predicted by urban habitat heterogeneity. These

234 additional meta-analyses, although limited by intrinsic features of the data set (see

235 Supplementary text 1) showed that, in our data set, urban habitats were spatially more

236 heterogeneous than non-urban habitats ${ }^{29}$ (Figure 3a) and that urban habitat heterogeneity

237 was associated with higher phenotypic variation in urban bird populations compared to their

238 non-urban counterparts, especially at large spatial scales (Figure 3b; see Supplementary

239 text 1 for full details and discussion of the limitations of this complementary analysis). Our

240 findings do not suffer from common publications biases, small-study effects or time-lag

241 effects (Supplementary text 2). 

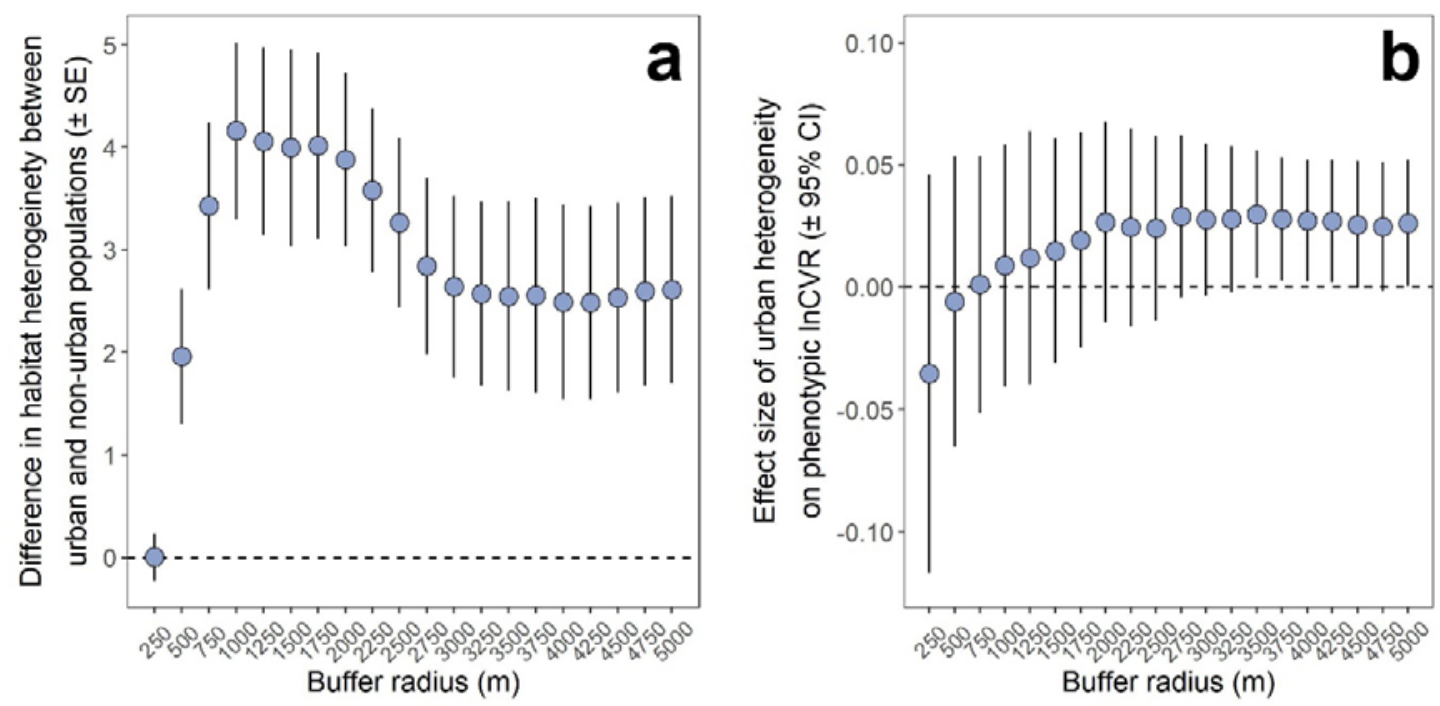

Figure 3. Urban heterogeneity effects on the difference in variation between urban and non-urban bird populations (i.e., InCVR). (a) Habitat heterogeneity was higher in urban populations than in non-urban populations across most spatial scales (e.g., different buffer sizes). (b) The higher the difference in habitat heterogeneity between urban and non-urban populations, the higher the difference in phenotypic variation between urban and non-urban populations tended to be (i.e., positive effect of urban heterogeneity on InCVR); particularly at a relatively large spatial scale (e.g., buffer radius larger than $2000 \mathrm{~m}$ ). Points represent (a) mean difference in habitat heterogeneity between urban and non-urban populations $( \pm S E)$, and (b) the effect size of urban habitat heterogeneity on the difference in phenotypic variation across habitats (i.e., mean InCVR and its 95\% confidence interval). 'Buffer radius' refers to the radius of a circular area centred at each study location and over which habitat heterogeneity was calculated (see Supplementary text 1 for more details). 
265 Table 1. Differences in variation (InCVR) in life-history traits between urban and non-

266 urban populations at different temporal scales. Urban - non-urban differences in

267 variation (InCVR) in laying dates, clutch size and number of fledglings per clutch were meta-

268 analysed to assess differences in variation between urban and non-urban populations within

269 and between breeding seasons (e.g., different temporal scales). InCVR estimates represent

270 meta-analytic model intercepts following the model structure presented in Table S5; positive

271 values indicate higher variation in urban populations than in non-urban populations and vice

272 versa. ' $\mathrm{Cl}$ ' = confidence interval; ' $\mathrm{k}$ ' = sample size. Terms in italic bold highlight InCVR

273 estimates whose $95 \%$ Cls do not overlap zero.

\begin{tabular}{|c|c|c|c|c|}
\hline \multirow{2}{*}{ Temporal scale } & \multicolumn{3}{|c|}{ InCVR estimate $[95 \% \mathrm{Cl}]$} & \multirow{2}{*}{$\mathbf{k}$} \\
\hline & Laying date & Clutch size & Number of fledglings & \\
\hline Overall & $\begin{array}{c}0.193 \\
{[0.107,0.279]}\end{array}$ & $\begin{array}{c}0.0518 \\
{[-0.046,0.149]}\end{array}$ & $\begin{array}{c}0.0635 \\
{[-0.071,0.198]}\end{array}$ & 409 \\
\hline $\begin{array}{c}\text { Within breeding } \\
\text { season }\end{array}$ & $\begin{array}{c}0.193 \\
{[0.094,0.292]}\end{array}$ & $\begin{array}{c}0.024 \\
{[-0.110,0.158]}\end{array}$ & $\begin{array}{c}0.149 \\
{[-0.028,0.322]}\end{array}$ & 376 \\
\hline $\begin{array}{c}\text { Between breeding } \\
\text { seasons }\end{array}$ & $\begin{array}{c}0.007 \\
{[-0.133,0.147]}\end{array}$ & $\begin{array}{c}0.0774 \\
{[-0.034,0.189]}\end{array}$ & $\begin{array}{c}0.011 \\
{[-0.136,0.158]}\end{array}$ & 101 \\
\hline
\end{tabular}




\section{Conclusion}

276 Taken together, our results show that urbanisation impacts not only the mean phenotype of

277 avian populations but also the amount of phenotypic variation that these populations display.

278 Our results also highlight a temporal and spatial mechanism that could generate such

279 differences in phenotypic variation among urban and non-urban habitats. That urban avian

280 populations have more variable phenology than non-urban conspecifics within breeding

281 seasons (i.e., differences in phenology across habitats are seemingly not due to between-

282 year fluctuations) suggests that the opportunity for selection could be consistently higher in

283 urban environments than in non-urban environments, even for species in which most

284 individuals reproduce in a single breeding season only.

286 Why do urban populations display higher phenotypic variation that their non-urban

287 neighbours? Our analyses reveal this pattern and shed light on the temporal and spatial

288 scale at which it occurs but do not explain how it is generated. One possibility that our spatial

289 analysis highlights is that high environmental heterogeneity in urban habitats ${ }^{32}$ might allow

290 for plastic responses to microhabitat conditions, thereby increasing the amount of phenotypic

291 variation in urban bird populations ${ }^{16}$. Alternatively, higher phenological variation in urban

292 environments could be a consequence of relaxed selection in this habitat ${ }^{35}$ or gene flow from

293 non-urban to urban habitats ${ }^{36}$. Weaker selection for early laying in urban habitats has been

294 found in at least one bird species ${ }^{35}$, which coupled with the high dispersal ability of birds,

295 could increase the amount of phenotypic variation in phenology. The ecological and

296 evolutionary implications of higher phenotypic variation in urban environments will likely vary

297 among species $^{9}$ and our findings highlight the need for detailed investigation of these

298 consequences. To this end, long-term studies of individually marked individuals in paired

299 urban and non-urban environments could be particularly fruitful. 


\section{Methods}

\section{$301 \quad$ Literature review}

302 We began our literature search by inspecting two published reviews on the impact of 303 urbanisation on avian biology ${ }^{10,13}$. As we were mainly interested in how phenology and 304 reproduction were affected by urbanisation, we identified papers cited in Chamberlain et al. 305 (2009) $(\mathrm{N}=37)$ and Sepp et al. (2018) $(\mathrm{N}=32)$ that could contain estimates for egg laying dates (hereafter laying dates), clutch size and number of nestlings fledged per breeding attempt (hereafter number of fledglings) for urban and non-urban populations. Then, we performed four searches of the Web of Science Core Collection on the $27^{\text {th }}$ of October 2020 (databases covered: SCI-EXPANDED - 1900-present, SSCI - 1956-present, A\&HCI - 1975present, BKCl-S - 2005-present, BKCl-SSH - 2005-present and ESCI - 2015-present) to recover studies published since 1900 and including all languages and all document types.

312 We performed the following four searches: (1) TS=("urban*" AND ("bird" OR "aves" OR "avian" OR "ornithol*" OR "passerine*" OR "passeriform*" OR "songbird*" OR list of bird genera) AND ("laying date" OR "lay date" OR "first egg" OR "clutch size" OR "eggs laid" OR "number of eggs" OR "fledgling*" OR "fledging" OR "reproductive success" OR "fitness")); (2) TS=("urban*" AND "bird" AND "laying date"); (3) TS=("urban*" AND "bird" AND "clutch size"); (4) TS=("urban*" AND "bird" AND "fledglings"). The list of avian genera in the first search string consisted of a list of all avian genera and can be found in Supplementary text 3 (this search string was created by Antje Girndt - see acknowledgments). Searches 2-4 were contained within search 1; however, they were not restricted to avian genera, hence potentially capturing additional references that did not mention the scientific name of the study species. These searches found 892, 71, 198 and 167 studies, respectively, which we combined with the studies identified from Chamberlain et al. (2009) and Sepp et al. (2018) to create a list of 1,397 studies (Figure S1). We then de-duplicated this list using the R package

325 'revtools' (using exact matching of study titles in function 'find_duplicates'; v0.4.137) and by 326 manually inspecting all study titles and author lists. Our final list contained 940 unique 
327 studies (Figure S1). We screened each study by reading their title and abstract. If the title

328 and/or abstract indicated that the paper could fit our requirements for data collection (see

329 below), we read the study fully, aiming to extract mean, standard deviation (SD) and sample

330 size $(n)$ of our life-history traits of interest for urban and non-urban bird populations. If SD

331 was not available but authors provided SE, the former was calculated as: $S D=S E \times \sqrt{n}$.

332 When available, we extracted estimates per year (i.e., papers often reported mean, SD and

$333 \mathrm{n}$ for urban and non-urban populations in multiple years of study). If a study reported

334 incomplete information for inclusion in our meta-analysis (e.g., mean was provided but not

335 SD or SE), we contacted the authors to ask for this missing information (a complete list of

336 authors that provided estimates can be found in the acknowledgments).

\section{Criteria for inclusion}

339 We were interested in investigating the effects of urbanisation on life-history traits, with a 340 particular interest in testing the effects of urbanisation on variation and mean trait values.

341 Paired urban - non-urban designs, where an urban population is compared to a non-urban 342 adjacent population are a powerful approach to identify the effects of urban living while 343 controlling for temporal and geographical variation, as well as large-scale genetic structure 344 among populations. Therefore, we included studies as long as they (i) compared 345 geographically close (i.e., paired) urban and non-urban populations and (ii) reported laying 346 dates of the first clutch of the season, clutch size or number of nestlings for the same 347 breeding season across both habitats. When multiple populations were compared along a 348 gradient of urbanisation, we extracted estimates for the two populations at the extremes of 349 the gradient (i.e. most urban and least urban populations). When studies combined 350 estimates across several breeding seasons, we included them in our meta-analysis as long 351 as urban and non-urban populations had been sampled in the same breeding seasons. We 352 found variation in the way that authors reported our three traits of interest. For example, 'egg 353 laying date' often referred to the date on which clutches were completed, but sometimes this 354 value indicated the laying date of the first egg of the clutch. Sometimes authors did not 
355 specify if by 'laying dates' they referred to clutch completion dates or first egg laying dates.

356 We always tried to extract information on the number of fledglings considering all breeding

357 attempts regardless of whether none or some nestlings survived in a given attempt.

358 However, studies sometimes reported number of fledglings across habitats considering only

359 successful broods (i.e., those that fledged at least one nestling). Although there may be

360 subtle methodological differences among some of the studies included in our data set,

361 among-study variation is not likely to bias results since our effect sizes (see below) consist of

362 within-study comparisons where the methods were the same for urban and non-urban 363 populations.

Initially, our data set contained 427 urban - non-urban paired estimates from 33 bird species and 65 studies. Of these, three observations were removed due to missing sample sizes, and 11 observations were removed because their sample size was one (which precludes the calculation of a mean and their SD). Four observations were removed because they reported a SD of zero (these indeed had very low sample sizes: 3, 2, 7, 2 observations). One effect size for laying dates, nine effect sizes for clutch size and 13 effect sizes for number of fledglings from five studies contained missing SD data. Mean and SD are often strongly correlated $^{38}$, including in our dataset (Figure S3). Therefore, we imputed the 23 missing SD values based on linear regressions of log mean against log standard deviation. Our final data set included 409 comparisons between urban and non-urban populations from 65

375 published papers and 33 bird species (Figure 1; refs. ${ }^{26,27,35,39-100}$ ). Of these 409 comparisons, 151 corresponded to comparisons of laying dates ( $\mathrm{N}=32$ studies), 124 corresponded to comparisons of clutch size ( $N=42$ studies) and 134 were comparisons of number of fledglings ( $N=44$ studies). Last, there were a total of 376 comparisons for single years ( $N=47$ studies) and an additional 33 comparisons included estimates across multiple years of study ( $\mathrm{N}=18$ studies). 


\section{Coding of effect sizes}

385 Laying date data was standardised across studies by coding it as days after January 1 . In every case, mean laying date estimates across habitats fell within the same calendar year. For each of these three life-history traits we computed the log response ratio (InRR) and the log coefficient of variation ratio (InCVR) to investigate differences in mean values and variability between urban and non-urban populations ${ }^{18,19}$. We calculated InRR and InCVR along with their associated sampling variances ${ }^{18}$ using the $\mathrm{R}$ function 'escalc' in the 'metafor' R package (v2.5-57; Viechtbauer 2010). Both InRR and InCVR were coded in a way that positive values meant higher estimates in urban populations compared to their non-urban counterparts. Urban and non-urban populations can differ in mean and variance levels at the same time (e.g., Taylor's Law ${ }^{38,102}$ ), as observed in our data (Figure S3). Therefore, we chose InCVR over InVR (i.e., log total variation ratio ${ }^{18}$ ) as the former is designed to account for the mean-variance relationship ${ }^{18,19}$. We handled the data set, ran all analyses and produced visualisations using R v.4.0.3 ${ }^{103}$.

\section{Meta-analyses and random effects}

To evaluate the effects of urbanisation on avian life-history traits, we fitted phylogenetic multilevel (intercept-only) meta-analyses for each response term (i.e. InCVR or InRR) combining all three life-history traits (i.e. laying date, clutch size and number of fledglings). All These meta-analytic models estimated five random intercept effects: publication identity

404 (i.e., among-study variation), population identity, phylogeny (more details below) and species identity (i.e., among-species variation not explained by phylogeny), as well as a residual variance and/or within-study variation. We estimated total heterogeneity $(\mathcal{P})$ and the proportion of variation explained by each random term in our meta-analytic (intercept-only) models following ${ }^{21,104}$ and as implemented in the R function 'i2_ml' ('metafor' R package v2.5-5 $\left.57^{101}\right)$. 


\section{Modelling heterogeneous variances and correlations among traits}

414 Laying date, clutch size and number of fledglings are often correlated in bird species ${ }^{23}$. To assess the robustness of our results to the existence of these correlations in our data set, we

416 built meta-analytic models that allowed us to simultaneously estimate trait-specific means 417 (i.e., one intercept for each trait - Equation 1), trait-specific residual variances (i.e., one 418 'observation identity' variance for each trait - Equation 1 \& Equation 2) and trait-specific 419 among-study variances and correlation among traits (Equation 1 \& Equation 3). Including the 420 random-effect terms detailed above, our model with heterogeneous variances and amongstudy correlations among traits can be written as: (we have omitted the term associated with sampling variance for simplicity - see Nakagawa et al. (2015) for more details)

$$
y_{i}=\left[\begin{array}{c}
\mu_{L D} \\
\mu_{C S} \\
\mu_{N F}
\end{array}\right]+\left[\begin{array}{c}
\varepsilon_{i-L D} \\
\varepsilon_{i-C S} \\
\varepsilon_{i-N F}
\end{array}\right]+\left[\begin{array}{c}
\tau_{t-L D} \\
\tau_{t-C S} \\
\tau_{t-N F}
\end{array}\right]+v_{a}+a_{l}+h_{w} \text { (Equation 1) }
$$

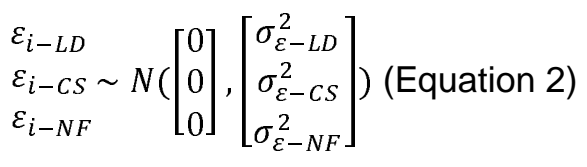

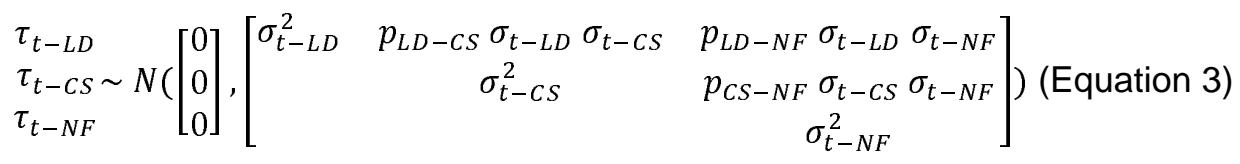

$$
v_{a} \sim N\left(0, \sigma_{v}^{2}\right) \text { (Equation 4) }
$$

$$
a_{l} \sim N\left(0, A \sigma_{a}^{2}\right)(\text { Equation 5) }
$$

$$
h_{w} \sim N\left(0, \sigma_{h}^{2}\right) \text { (Equation 6) }
$$


436 Where $y_{i}$ is the statistic of interest (InRR or $\left.\operatorname{lnCVR}\right)$ for the $i$ th effect size $(i=1,2,3, \ldots, k$;

437 where $k$ is the number of the effect sizes included in the analysis - i.e., number of urban -

438 non-urban paired comparisons). 'LD', 'CS', 'NF' refer to overall means $(\mu)$, variances $(\sigma)$ and

439 correlations $(\rho)$ involving effect sizes for laying date ('LD'), clutch size ('CS') and number of

440 fledglings ('NF'). $\varepsilon_{i}$ is the residual deviation for the $i$ th observation, which is assumed to

441 follow a normal distribution with mean 0 and variance $\sigma_{\varepsilon-L D}^{2}, \sigma_{\varepsilon-C S}^{2}, \sigma_{\varepsilon-N F}^{2}$ for laying date,

442 clutch size and number of fledglings, respectively. $\tau_{t-L D}, \tau_{t-C S}$, and $\tau_{t-N F}$ are the deviations

443 from the mean associated with the $t$ th study and trait ('LD', 'CS', or 'NF'), each following a

444 multivariate normal distribution with mean of 0 and variance-covariance structure detailed in

445 Equation 5 ( $p$ provides the correlations between $\tau_{t-L D}, \tau_{t-C S}$, and $\tau_{t-N F}$ ). $v_{a}$ provides the

446 deviation from the overall mean associated with the ath population. $a_{l}$ is the phylogenetic

447 effect for the $l$ th species, which follows a normal distribution with mean equal to 0 and

448 variance-covariance structure given by $\sigma_{a}^{2}$, the variance of the phylogenetic effect, and $A$, a $l$

449 by $l$ matrix of distances between species calculated from a phylogenetic tree (more details

450 below). $h_{w}$ captures among species variation not explained by the phylogenetic effect and

451 follows a normal distribution around 0 and variance $\sigma_{h}^{2}$.

453 We compared models with different constraints in the parameters of the variance-covariance

454 structure in Equation 3 to assess the strength of evidence for heterogeneous variances and

455 correlations among traits. We fitted the meta-analytic models in the 'metafor' R package

456 (v2.5-57; Viechtbauer 2010) using maximum likelihood and compared model AIC (Akaike

457 information criterion ${ }^{105}$ ). We then calculated a $\triangle$ AIC value for each model (i.e., the difference

458 in AIC between a given model and the model with lowest AIC) and used this value to assess

459 the strength of evidence for a given variance-covariance structure. We fitted models with the

460 following constraints in the variance-covariance structure:

461 (i) Single variance across traits and no correlations: 


$$
\sigma_{t-L D}^{2}=\sigma_{t-C S}^{2}=\sigma_{t-N F}^{2} ; \text { and all } p=0
$$

(ii) Compound symmetric variance-covariance matrix:

$$
\sigma_{t-L D}^{2}=\sigma_{t-C S}^{2}=\sigma_{t-N F}^{2} ; \text { and } p_{L D-C S}=p_{L D-N F}=p_{C S-N F}
$$

(iii) Heteroscedastic compound symmetric variance-covariance matrix:

$$
\sigma_{t-L D}^{2}, \sigma_{t-C S}^{2} \text { and } \sigma_{t-N F}^{2} \text { can vary freely but } p_{L D-C S}=p_{L D-N F}=p_{C S-N F}
$$

(iv) Diagonal variance-covariance matrix:

$$
\sigma_{t-L D}^{2}, \sigma_{t-C S}^{2} \text { and } \sigma_{t-N F}^{2} \text { can vary freely but all } p=0
$$

(v) Unstructured variance-covariance matrix $\sigma_{t-L D}^{2}, \sigma_{t-C S}^{2}$ and $\sigma_{t-N F}^{2}$ can vary freely, as well as $p_{L D-C S}, p_{L D-N F}$ and $p_{C S-N F}$ (i.e. correlation among traits).

\section{Within- and between-breeding season differences in phenology and life-history traits}

\section{between urban and non-urban population}

Urban and non-urban populations may differ in both within-breeding season and betweenbreeding season variation in phenology and life-history traits. However, differences in variation for these two temporal scales are likely generated by contrasting ecological and evolutionary processes ${ }^{9}$. To disentangle processes operating at these two temporal scales that generate differences in phenotypic variation between urban and non-urban populations, we performed additional meta-analyses including 1) urban - non-urban comparisons within breeding seasons ( $k=376$ comparisons in the original data set) and 2) urban - non-urban comparisons between breeding seasons (i.e., combining all within-breeding season estimates from a given study; $k=33$ between-breeding season comparisons present in the original data set, plus 68 additional comparison calculated from within-breeding season estimates; see below). When a given study reported estimates across multiple breeding seasons, we calculated between-breeding season mean and variance as:

$$
\bar{x}_{\text {among-season }}=\sum_{i=1}^{g} \bar{x}_{i} \frac{n_{i}}{N} ; \text { where }, N=\sum_{i=1}^{g} n_{i} \text { (Equation 10) }
$$




$$
S_{\text {among-season }}^{2}=\sum_{i=1}^{g} n_{i} s_{i}^{2}+\sum_{i=1}^{g} n_{i}\left(\bar{x}_{i}-\bar{x}_{\text {among-season }}\right)^{2}(\text { Equation 11) }
$$

491

Where, $\bar{x}_{\text {among-season }}$ and $S_{\text {among-season }}^{2}$ are mean and variance across multiple breeding

seasons. $g$ is the total number of breeding seasons reported by a given study; $\bar{x}_{i}, s_{i}^{2}, n_{i}$, are

mean, variance and sample size for each breeding season $i . \bar{x}_{\text {among-season }}$ for a given study

is, therefore, the weighted average across breeding seasons (Equation 10); whereas

$S_{\text {among-season }}^{2}$ for a given study is the weighted sum of within-season variances (first term in

equation 11) and between-season mean variances (second term in Equation 11).

\section{Phylogenies}

500 Phylogenetic trees were extracted from the Open Tree of Life ${ }^{106,107}$, using the interface provided by the R package 'rotl' (v3.0.10; Michonneau et al. 2016, OpenTreeOfLife et al. 2019). We calculated tree branch length ${ }^{110}$ and generated a phylogenetic correlation matrix to include in our (comparative) meta-analytic models (Figure 1). We assessed phylogenetic signal in our meta-analysis based on the proportion of variation explained by the phylogeny

\section{Publication bias}

508 We assessed the existence of two types of publication biases, small-study and time-lag 509 effects, following the recommendation in ${ }^{106}$. We ran our meta-models for InRR and InCVR 510 including two new moderators: 1) the square-root of the inverse of the effective sample size 511 and 2) the year of study publication (mean-centered) ${ }^{111,112}$. The statistical significance of 512 these two moderators provides evidence for the existence of small-study effects and time-lag 513 effects, respectively ${ }^{112}$. In order to assess the effect of the existence of small-study effect in 514 our estimates of $\ln R R$ and $\ln C V R$, we ran additional $\ln R R$ and $\ln C V R$ meta-regressions 
bioRxiv preprint doi: https://doi.org/10.1101/2021.09.24.461498; this version posted September 24, 2021. The copyright holder for this preprint (which was not certified by peer review) is the author/funder, who has granted bioRxiv a license to display the preprint in perpetuity. It is made available under aCC-BY-NC 4.0 International license.

515 including the inverse of the effective sample size as a moderator to estimate adjusted $\operatorname{lnRR}$

516 and InCVR intercepts for laying dates, clutch size and number of fledglings ${ }^{106}$. 


\section{Acknowledgments}

518 We would like to thank Paul Bellamy, Clint Boal, Peter Ferns, Michał Glaqdalski, Shelley A.

519 Hinsley, Piotr Minias, Christy Morrissey, Matthew Reudink, Staffan Roos, Renaud Scheifler,

520 Christine Stracey, Daniel Shustack and Jarosław Wawrzyniak for replying to our data

521 queries. We are grateful to Antje Girndt for developing the list of avian genera included in our

522 search string.

523

524 Funding

525 PC-L, CJB and DMD were funded by a Highlight Topics grant from the Natural Environment

526 Research Council awarded to DD (NE/S005773/1).

527

528 Author contributions

529 PC-L, MJT, DR, AC and DMD conceived the study. PC-L, AS-T, CJB and DMD performed

530 the literature search and PC-L extracted effect sizes from suitable published papers. PC-L

531 and $\mathrm{YH}$ performed all statistical analysis with advice form AS-T. PC-L wrote the first draft of

532 the manuscript with input from MJT, AS-T, DR, AC and DMD. All authors read and 533 commented the manuscript. 


\section{References}

537 1. Hendry, A. P., Gotanda, K. M. \& Svensson, E. I. Human influences on

538 evolution, and the ecological and societal consequences. Philos. Trans. $R$.

539 Soc. B 372, 20160028 (2017).

540 2. Grimm, N. B. et al. Global change and the ecology of cities. Science 319, 756-

541760 (2008).

542 3. Johnson, M. T. J. \& Munshi-South, J. Evolution of life in urban environments.

$543 \quad$ Science 358, eaam8327 (2017).

544 4. Lambert, M. R., Brans, K. I., Des Roches, S., Donihue, C. M. \& Diamond, S. E.

$545 \quad$ Adaptive Evolution in Cities: Progress and Misconceptions. Trends Ecol. Evol.

$546 \quad 36,239-257$ (2020).

547 5. Alberti, M. et al. Global urban signatures of phenotypic change in animal and

6. Diamond, S. E., Chick, L. D., Perez, A., Strickler, S. A. \& Martin, R. A.

Evolution of thermal tolerance and its fitness consequences: parallel and nonparallel responses to urban heat islands across three cities. Proc. R. Soc. $B$ 285, 20180036 (2018).

8. Diamond, S. E. \& Martin, R. A. Evolution in Cities. Annu. Rev. Ecol. Evol. Syst. 52, 519-540 (2021).

9. Thompson, M. J., Capilla-Lasheras, P., Dominoni, D. M., Réale, D. \&

Charmantier, A. Phenotypic variation in urban environments: mechanisms and implications. In prep.

10. Sepp, T., McGraw, K. J., Kaasik, A. \& Giraudeau, M. A review of urban impacts on avian life-history evolution: Does city living lead to slower pace of life? Glob. Chang. Biol. 24, 1452-1469 (2018).

11. Sanders, D., Frago, E., Kehoe, R., Patterson, C. \& Gaston, K. J. Impacts of Artificial Light at Night on Biological Timings. Nat. Ecol. Evol. 68 (2020).

12. Putman, B. J. \& Tippie, Z. A. Big City Living: A Global Meta-Analysis Reveals Positive Impact of Urbanization on Body Size in Lizards. Front. Ecol. Evol. 8, 580745 (2020).

13. Chamberlain, D. E., Cannon, A. R., Toms, M. P. \& Leech, D. I. Avian 
570 productivity in urban landscapes $\square$ : a review and meta-analysis. Ibis 151, 1-18

571 (2009).

572 14. Vaugoyeau, M. et al. Interspecific variation in the relationship between clutch size, laying date and intensity of urbanization in four species of hole-nesting birds. Ecol. Evol. 6, 5907-5920 (2016).

15. Fugère, V. \& Hendry, A. P. Human influences on the strength of phenotypic selection. Proc. Natl. Acad. Sci. U. S. A. 115, 10070-10075 (2018).

16. Gorton, A. J., Moeller, D. A. \& Tiffin, P. Little plant, big city: A test of adaptation to urban environments in common ragweed (Ambrosia artemisiifolia). Proc. $R$. Soc. B 285, 20180968 (2018).

17. Hedges, L., Gurevitch, J. \& Curtis, P. The Meta-Analysis of Response Ratios in Experimental Ecology. Ecology 80, 1150-1156 (1999).

18. Nakagawa, S. et al. Meta-analysis of variation: Ecological and evolutionary applications and beyond. Methods Ecol. Evol. 6, 143-152 (2015).

19. Senior, A. M., Viechtbauer, W. \& Nakagawa, S. Revisiting and expanding the meta-analysis of variation: The log coefficient of variation ratio. Res. Synth. Methods 1-15 (2020).

20. Cinar, O., Nakagawa, S. \& Viechtbauer, W. hylogenetic multilevel metaanalysis: A simulation study on the importance of modeling the phylogeny. EcoEvoRxiv (2020).

21. Nakagawa, S. \& Santos, E. S. A. Methodological issues and advances in biological meta-analysis. Evol. Ecol. 26, 1253-1274 (2012).

22. Sánchez $\square$ Tójar, A., Moran, N. P., O’Dea, R. E., Reinhold, K. \& Nakagawa, S. Illustrating the importance of meta $\square$ analysing variances alongside means in ecology and evolution. J. Evol. Biol. 33, 1216-1223 (2020).

23. Dunn, P. O. \& Møller, A. P. Changes in breeding phenology and population size of birds. J. Anim. Ecol. 83, 729-739 (2014).

24. Garant, D., Hadfield, J. D., Kruuk, L. E. B. \& Sheldon, B. C. Stability of genetic variance and covariance for reproductive characters in the face of climate change in a wild bird population. Mol. Ecol. 17, 179-188 (2008).

25. Dominoni, D., Quetting, M. \& Partecke, J. Artificial light at night advances avian reproductive physiology. Proc. $R$. Soc. B 280, 20123017 (2013).

26. Seress, G. et al. Impact of urbanization on abundance and phenology of caterpillars and consequences for breeding in an insectivorous bird. Ecol. 
604

605

606

607

608

Appl. 28, 1143-1156 (2018).

27. Seress, G., Sándor, K., Evans, K. L. \& Liker, A. Food availability limits avian reproduction in the city: An experimental study on great tits Parus major. J. Anim. Ecol. 89, 1570-1580 (2020).

28. Downhower, J. F., Blumer, L. S. \& Brown, L. Opportunity for selection: An appropriate measure for evaluating variation in the potential for selection? Evolution 41, 1395-1400 (1987).

29. Pickett, S. T. A. et al. Dynamic heterogeneity: a framework to promote ecological integration and hypothesis generation in urban systems. Urban Ecosyst. 20, 1-14 (2017).

30. Heisler, J. M. \& Brazel, A. J. The urban physical environment: temperature and urban heat islands. in Urban Ecosystem Ecology (eds. Aitkenhead-Petersn, J. \& Volder, A.) 29-56 (American Society of Agronomy, Crop Science Society of America, Soil Science Society of America, 2018).

31. Strubbe, D. et al. Phenotypic signatures of urbanization are scale-dependent: A multi-trait study on a classic urban exploiter. Landsc. Urban Plan. 197, 103767 (2020).

32. Shochat, E., Warren, P. S., Faeth, S. H., Mclntyre, N. E. \& Hope, D. From patterns to emerging processes in mechanistic urban ecology. Trends Ecol. Evol. 21, 186-191 (2006).

33. Arnfield, A. J. Two decades of urban climate research: A review of turbulence, exchanges of energy and water, and the urban heat island. Int. J. Climatol. 23, 1-26 (2003).

34. Rodewald, A. D. \& Arcese, P. Reproductive contributions of cardinals are consistent with a hypothesis of relaxed selection in urban landscapes. Front. Ecol. Evol. 5, 77 (2017).

35. Caizergues, A. E., Charmantier, A. \& Grégoire, A. Urban versus forest ecotypes are not explained by divergent reproductive selection. Proc. $R$. Soc. $B$ 285, 20180261 (2018).

36. Salmón, P. et al. Continent-wide genomic signatures of adaptation to urbanisation in a songbird across Europe. Nat. Commun. 12, 2983 (2021).

37. Westgate, M. J. revtools: An R package to support article screening for evidence synthesis. Res. Synth. Methods 10, 606-614 (2019).

38. Nakagawa, S. \& Schielzeth, H. A general and simple method for obtaining R2 
from generalized linear mixed-effects models. Methods Ecol. Evol. 4, 133-142 (2013).

39. Brahmia, Z. et al. Breeding performance of blue tits (Cyanistes cæruleus ultramarinus) in relation to lead pollution and nest failure rates in rural, intermediate, and urban sites in Algeria. Environ. Pollut. 174, 171-178 (2013).

40. Capilla-Lasheras, P. et al. Elevated Immune Gene Expression Is Associated with Poor Reproductive Success of Urban Blue Tits. Front. Ecol. Evol. 5, 64 (2017).

41. Cardilini, A. P. A., Weston, M. A., Nimmo, D. G., Dann, P. \& Sherman, C. D. H. Surviving in sprawling suburbs: Suburban environments represent high quality breeding habitat for a widespread shorebird. Landsc. Urban Plan. 115, 72-80 (2013).

42. Conway, C. J., Garcia, V., Smith, M. D., Ellis, L. A. \& Whitney, J. L. Comparative demography of Burrowing Owls in agricultural and urban landscapes in southeastern Washington. J. F. Ornithol. 77, 280-290 (2006).

43. de Satgé, J. et al. Urbanisation lowers great tit Parus major breeding success at multiple spatial scales. J. Avian Biol. e02108 (2019) doi:10.1111/jav.02108.

44. Dhondt, A. A., Eyckerman, R., Moermans, R. \& Hublé, J. Habitat and laying date of Great and Blue Tit Parus major and P. caeruleus. Ibis (Lond. 1859). 126, 388-397 (1984).

45. Eden, S. F. The comparative breeding biology of magpies Pica pica in an urban and a rural habitat (Aves: Corvidae). J. Zool. 205, 325-334 (1985).

46. Gahbauer, M. A. et al. Productivity, mortality, and management of urban peregrine falcons in northeastern North America. J. Wildl. Manage. 79, 10-19 (2015).

47. Glądalski, M. et al. Effects of extreme thermal conditions on plasticity in breeding phenology and double-broodedness of Great Tits and Blue Tits in central Poland in 2013 and 2014. Int. J. Biometeorol. 60, 1795-1800 (2016).

48. Glądalski, M. et al. Differences in the breeding success of blue tits Cyanistes caeruleus between a forest and an urban area: A long-term study. Acta Ornithol. 52, 59-68 (2017).

49. Glądalski, M. et al. Effects of nest characteristics on reproductive performance in Blue Tits Cyanistes caeruleus and Great Tits Parus major. Avian Biol. Res. 9, 37-43 (2016). 
672 50. Glądalski, M. et al. Inter-annual and inter-habitat variation in breeding

673 performance of blue tits (Cyanistes caeruleus) in central Poland. Ornis Fenn.

$67492,34-42(2015)$.

675 51. Gryz, J. \& Krauze-Gryz, D. Influence of habitat urbanisation on time of

52. Hajdasz, A. C., Otter, K. A., Baldwin, L. K. \& Reudink, M. W. Caterpillar breeding and productivity of tawny Owl (Strix aluco). Polish J. Ecol. 66, 153161 (2018). phenology predicts differences in timing of mountain chickadee breeding in urban and rural habitats. Urban Ecosyst. 22, 1113-1122 (2019).

53. Hinsley, S. A. et al. Effects of structural and functional habitat gaps on breeding woodland birds: Working harder for less. Landsc. Ecol. 23, 615-626 (2008).

54. Isaksson, C. \& Andersson, S. Carotenoid diet and nestling provisioning in urban and rural great tits Parus major. J. Avian Biol. 38, 564-572 (2007).

55. Isaksson, C., Johansson, A. \& Andersson, S. Egg yolk carotenoids in relation to habitat and reproductive investment in the great tit Parus major. Physiol. Biochem. Zool. 81, 112-118 (2008).

56. Jarrett, C., Powell, L. L., McDevitt, H., Helm, B. \& Welch, A. J. Bitter fruits of hard labour: diet metabarcoding and telemetry reveal that urban songbirds travel further for lower-quality food. Oecologia 193, 377-388 (2020).

57. Ibáñez-Álamo, J. D. \& Soler, M. Does urbanization affect selective pressures and life-history strategies in the common blackbird (Turdus merula L.)? Biol. J. Linn. Soc. 101, 759-766 (2010).

58. Kelleher, K. M. \& O'Halloran, J. Influence of nesting habitat on breeding Song Thrushes Turdus philomelos. Bird Study 54, 221-229 (2007).

59. Antonov, A. \& Atanasova, D. Y. Small-scale differences in the breeding ecology of urban and rural Magpies Pica pica. Ornis Fenn. 80, 21-30 (2003).

60. Kettel, E. F., Gentle, L. K., Yarnell, R. W. \& Quinn, J. L. Breeding performance of an apex predator, the peregrine falcon, across urban and rural landscapes. Urban Ecosyst. 22, 117-125 (2019).

61. Lee, S., Lee, H., Jablonski, P. G., Choe, J. C. \& Husby, M. Microbial abundance on the eggs of a passerine bird and related fitness consequences between urban and rural habitats. PLoS One 12, e0185411 (2017).

62. Lin, W. L., Lin, S. M., Lin, J. W., Wang, Y. \& Tseng, H. Y. Breeding 
performance of Crested Goshawk Accipiter trivirgatus in urban and rural environments of Taiwan. Bird Study 62, 177-184 (2015).

63. Liven-Schulman, I., Leshem, Y., Alon, D. \& Yom-Tov, Y. Causes of population declines of the Lesser Kestrel Falco naumanni in Israel. Ibis (Lond. 1859). 146, 145-152 (2004).

64. Luna, Á., Palma, A., Sanz-Aguilar, A., Tella, J. L. \& Carrete, M. Sex, personality and conspecific density influence natal dispersal with lifetime fitness consequences in urban and rural burrowing owls. PLoS One 15, 1-17 (2020).

65. Mennechez, G. \& Clergeau, P. Effect of urbanisation on habitat generalists: starlings not so flexible? Acta Oecologica 30, 182-191 (2006).

66. Meyrier, E. et al. Happy to breed in the city? Urban food resources limit reproductive output in Western Jackdaws. Ecol. Evol. 7, 1363-1374 (2017).

67. Middleton, A. Influence of Age and Habitat on Reproduction by the American Goldfinch. Ecology 60, 418-432 (1979).

68. Millsap, B. et al. Comparative Fecundity and Survival of Bald Eagles Fledged from Suburban and Rural Natal Areas in Florida. J. Wildl. Manage. 68, 10181031 (2004).

69. Minias, P. Reproduction and survival in the city: Which fitness components drive urban colonization in a reed-nesting waterbird? Curr. Zool. 62, 79-87 (2016).

70. Bailly, J. et al. From eggs to fledging: Negative impact of urban habitat on reproduction in two tit species. J. Ornithol. 157, 377-392 (2016).

71. Morrissey, C. A. et al. Developmental impairment in eurasian dipper nestlings exposed to urban stream pollutants. Environ. Toxicol. Chem. 33, 1315-1323 (2014).

72. Newhouse, M. J., Marra, P. P. \& Johnson, L. S. Reproductive Success of House Wrens in Suburban and Rural Landscapes. Wilson J. Ornithol. 120, 99104 (2008).

73. Pande, S., Pawashe, A., Mahajan, M. N., Joglekar, C. \& Mahabal, A. Effect of food and habitat on breeding success in Spotted Owlets (Athene brama) nesting in villages and rural landscapes in India. J. Raptor Res. 41, 26-34 (2007).

74. Partecke, J., Hegyi, G., Fitze, P. S., Gasparini, J. \& Schwabl, H. Maternal 
effects and urbanization: Variation of yolk androgens and immunoglobulin in city and forest blackbirds. Ecol. Evol. 10, 2213-2224 (2020).

75. Perlut, N. G., Bonter, D. N., Ellis, J. C. \& Friar, M. S. Roof-Top Nesting in a Declining Population of Herring Gulls (Larus argentatus) in Portland, Maine, USA. Waterbirds 39, 68-73 (2016).

76. Perrins, C. M. Population Fluctuations and Clutch-Size in the Great Tit. J. Anim. Ecol. 34, 601-647 (1965).

77. Pollock, C. J., Capilla-Lasheras, P., McGill, R. A. R., Helm, B. \& Dominoni, D. M. Integrated behavioural and stable isotope data reveal altered diet linked to low breeding success in urban-dwelling blue tits (Cyanistes caeruleus). Sci. Rep. 7, 5014 (2017).

78. Preiszner, B. et al. Problem-solving performance and reproductive success of great tits in urban and forest habitats. Anim. Cogn. 20, 53-63 (2017).

79. Rollinson, D. J. \& Jones, D. N. Variation in breeding parameters of the Australian magpie Gymnorhina tibicen in suburban and rural environments. Urban Ecosyst. 6, 257-269 (2003).

80. Rosenfield, R. N., Hardin, M. G., Taylor, J., Sobolik, L. E. \& Frater, P. N. Nesting density and dispersal movements between urban and rural habitats of cooper's Hawks (Accipiter cooperii) in Wisconsin: Are these source or sink habitats? Am. Midl. Nat. 182, 36-51 (2019).

81. Beck, N. R. \& Heinsohn, R. Group composition and reproductive success of cooperatively breeding white-winged choughs ( Corcorax melanorhamphos) in urban and non-urban habitat. Austral Ecol. 31, 588-596 (2006).

82. Schmidt, V. K.-H. \& Steinbach, J. Niedriger Bruterfolg der Kohlmeise(Parus major) in städtischen Parks und Friedhöfen. J. für Ornithol. 124, 81-83 (1983).

83. Schoech, S. J. \& Bowman, R. Variation in the timing of breeding between suburban and wildland Florida Scrub-Jays: Do physiologic measures reflect different environments? in Avian Ecology and Conservation in an Urbanizing World (eds. Marzluff, J. M., Bowman, R. \& Donelly, R.) 289-306 (Kluwer Academic Press, 2001).

84. Schoech, S. J., Bowman, R., Bridge, E. S. \& Boughton, R. K. Baseline and acute levels of corticosterone in Florida Scrub-Jays (Aphelocoma coerulescens): Effects of food supplementation, suburban habitat, and year. Gen. Comp. Endocrinol. 154, 150-160 (2007). 
774 85. Seress, G. et al. Urbanization, nestling growth and reproductive success in a

89. Solonen, T. \& Ursin, K. A. Breeding of Tawny Owls Strix aluco in rural and urban habitats in southern Finland. Bird Study 55, 216-221 (2008).

90. Stout, W. E., Anderson, R. K. \& Papp, J. M. Urban, suburban and rural redtailed hawk nesting habitat and populations in southeast Wisconsin. J. Raptor Res. 32, 221-228 (1998).

91. Stracey, C. M. \& Robinson, S. K. Are urban habitats ecological traps for a native songbird? Season-long productivity, apparent survival, and site fidelity in urban and rural habitats. J. Avian Biol. 43, 50-60 (2012).

92. Berardelli, D., Desmond, M. J. \& Murray, L. Reproductive success of burrowing owls in urban and grassland habitats in southern New Mexico. Wilson J. Ornithol. 122, 51-59 (2010).

93. Sumasgutner, P., Nemeth, E., Tebb, G., Krenn, H. W. \& Gamauf, A. Hard times in the city-attractive nest sites but insufficient food supply lead to low reproduction rates in a bird of prey. Front. Zool. 34, 17-31 (2014).

94. Thornton, M., Todd, I. \& Roos, S. Breeding Success and Productivity of Urban and Rural Eurasian Sparrowhawks Accipiter nisus in Scotland. Ecoscience 24, 115-116 (2017).

95. Wawyrzyniak, J. et al. Long-Term Variation in Laying Date and Clutch Size of the Great Tit Parus major in Central Poland: A Comparison between Urban Parkland and Deciduous Forest. Ardeola 62, 311-322 (2015).

96. Welch-Acosta, B. C., Skipper, B. R. \& Boal, C. W. Comparative breeding ecology of Mississippi Kites in urban and exurban areas of West Texas. J. F. Ornithol. 90, 248-257 (2019). 
97. Mcgowan, K. J. Avian Ecology and Conservation in an Urbanizing World. in Avian Ecology and Conservation in an Urbanizing World (eds. Marzluff, J. M., Bowman, R. \& Donelly, R.) 365-381 (Kluwer Academic Press, 2001).

98. Biard, C. et al. Growing in cities: An urban penalty for wild birds? A study of phenotypic differences between urban and rural great tit chicks (Parus major). Front. Ecol. Evol. 5, 79 (2017).

99. Boal, C. W. \& Mannan, R. . W. Comparative Breeding Ecology of Cooper's Hawks in Urban and Exurban Areas of Southeastern Arizona. J. Wildl. Manage. 63, 77-84 (1999).

100. Bobek, O., Gal, A., Saltz, D. \& Motro, U. Effect of nest-site microclimatic conditions on nesting success in the Lesser Kestrel Falco naumanni. Bird Study 65, 444-450 (2018).

101. Viechtbauer, W. Conducting meta-analyses in $\mathrm{R}$ with the metafor. J. Stat. Softw. 36, 1-48 (2010).

102. Cohen, J. E. \& Xu, M. Random sampling of skewed distributions implies Taylor's power law of fluctuation scaling. Proc. Natl. Acad. Sci. 112, 77497754 (2015).

103. R Core Team. R: A language and environment for statistical computing. $R$ Foundation for Statistical Computing, Vienna, Austria. (2020).

104. Senior, A. M. et al. Heterogeneity in ecological and evolutionary metaanalyses: its magnitude and implications. Ecology 97, 3293-3299 (2016).

105. Burnham, K. P., Anderson, D. R. \& Huyvaert, K. P. AIC model selection and multimodel inference in behavioral ecology $\square$ : some background , observations , and comparisons. Behav. Ecol. Sociobiol. 65, 23-35 (2011).

106. Rees, J. A. \& Cranston, K. Automated assembly of a reference taxonomy for phylogenetic data synthesis. Biodivers. Data J. 5, e12581 (2017).

107. Hinchliff, C. E. et al. Synthesis of phylogeny and taxonomy into a comprehensive tree of life. Proc. Natl. Acad. Sci. U. S. A. 112, 12764-12769 (2015).

108. Michonneau, F., Brown, J. W. \& Winter, D. J. rotl: an R package to interact with the Open Tree of Life data. Methods Ecol. Evol. 7, 1476-1481 (2016).

109. OpenTreeOfLife et al. Open Tree of Life Synthetic Tree. (2019) doi:10.5281/zenodo.3937741.

110. Grafen, A. The phylogenetic regression. Philos. Trans. R. Soc. London 326, 
119-157 (1989).

843 111. Trikalinos, T. \& loannidis, J. P. A. Assessing the evolution of effect sizes over time. in Publication Bias in Meta-Analysis (eds. Rothstein, H., Sutton, A. \& Borenstein, M.) 241-259 (John Wiley, 2005). doi:https://doi.org/ 10.1002/0470870168.ch13.

112. Nakagawa, S. et al. Methods for testing publication bias in ecological and evolutionary meta-analyses. EcoEvoRxiv (2021) doi:10.32942/osf.io/k7pmz. 